\title{
Inference of magnetic fields in the very quiet Sun
}

\author{
M. J. Martínez González ${ }^{1,2}$, A. Pastor Yabarr $^{1,2}$, A. Lagg $^{3}$, A. Asensio Ramos ${ }^{1,2}$, M. Collados ${ }^{1,2}$, S. K. Solanki ${ }^{3,4}$, \\ H. Balthasar ${ }^{5}$, T. Berkefeld ${ }^{6}$, C. Denker ${ }^{5}$, H. P. Doerr ${ }^{3}$, A. Feller ${ }^{3}$, M. Franz ${ }^{6}$, S. J. González Manrique ${ }^{5,7}$, \\ A. Hofmann ${ }^{5}$, F. Kneer ${ }^{8}$, C. Kuckein ${ }^{5}$, R. Louis ${ }^{5}$, O. von der Lühe ${ }^{6}$, H. Nicklas ${ }^{3}$, D. Orozco ${ }^{1,2}$, R. Rezaei ${ }^{1,2,6}$, \\ R. Schlichenmaier ${ }^{6}$, D. Schmidt ${ }^{6}$, W. Schmidt ${ }^{6}$, M. Sigwarth ${ }^{6}$, M. Sobotka ${ }^{9}$, D. Soltau ${ }^{6}$, J. Staude ${ }^{5}$, \\ K. G. Strassmeier ${ }^{5}$, M. Verma ${ }^{5}$, T. Waldman ${ }^{6}$, and R. Volkmer ${ }^{6}$ \\ 1 Instituto de Astrofísica de Canarias, Vía Láctea s/n, 38205 La Laguna, Tenerife, Spain \\ e-mail: marian@iac.es \\ 2 Dept. Astrofísica, Universidad de La Laguna, 38205 La Laguna, Tenerife, Spain \\ 3 Max-Planck-Institut für Sonnensystemforschung, Justus-von-Liebig Weg 3, 37077 Göttingen, Germany \\ ${ }^{4}$ School of Space Research, Kyung Hee University, Yongin, 446-701 Gyeonggi-Do, Republic of Korea \\ 5 Leibniz-Institut für Astrophysik Potsdam (AIP), An der Sternwarte 16, 14482 Potsdam, Germany \\ ${ }^{6}$ Kiepenheuer Institut für Sonnenphysik, Schöneckstr. 6, 79104 Freiburg, Germany \\ 7 Universität Potsdam, Institut für Physik und Astronomie, Karl-Liebknecht-Strasse 24/25, 14476 Potsdam-Golm, Germany \\ ${ }^{8}$ Institut für Astrophysik, Friedrich Hund Platz 1, 37077 Göttingen, Germany \\ 9 Astronomical Institute, Academy of Sciences of the Czech Republic, Fričova 298, 25165 Ondřejov, Czech Republic
}

Received 7 March 2016 / Accepted 6 July 2016

\begin{abstract}
Context. Over the past $20 \mathrm{yr}$, the quietest areas of the solar surface have revealed a weak but extremely dynamic magnetism occurring at small scales $(<500 \mathrm{~km})$, which may provide an important contribution to the dynamics and energetics of the outer layers of the atmosphere. Understanding this magnetism requires the inference of physical quantities from high-sensitivity spectro-polarimetric data with high spatio-temporal resolution.

Aims. We present high-precision spectro-polarimetric data with high spatial resolution $\left(0.4^{\prime \prime}\right)$ of the very quiet Sun at $1.56 \mu \mathrm{m}$ obtained with the GREGOR telescope to shed some light on this complex magnetism.

Methods. We used inversion techniques in two main approaches. First, we assumed that the observed profiles can be reproduced with a constant magnetic field atmosphere embedded in a field-free medium. Second, we assumed that the resolution element has a substructure with either two constant magnetic atmospheres or a single magnetic atmosphere with gradients of the physical quantities along the optical depth, both coexisting with a global stray-light component.

Results. Half of our observed quiet-Sun region is better explained by magnetic substructure within the resolution element. However, we cannot distinguish whether this substructure comes from gradients of the physical parameters along the line of sight or from horizontal gradients (across the surface). In these pixels, a model with two magnetic components is preferred, and we find two distinct magnetic field populations. The population with the larger filling factor has very weak $(\sim 150 \mathrm{G})$ horizontal fields similar to those obtained in previous works. We demonstrate that the field vector of this population is not constrained by the observations, given the spatial resolution and polarimetric accuracy of our data. The topology of the other component with the smaller filling factor is constrained by the observations for field strengths above $250 \mathrm{G}$ : we infer hG fields with inclinations and azimuth values compatible with an isotropic distribution. The filling factors are typically below $30 \%$. We also find that the flux of the two polarities is not balanced. From the other half of the observed quiet-Sun area $\sim 50 \%$ are two-lobed Stokes $V$ profiles, meaning that $23 \%$ of the field of view can be adequately explained with a single constant magnetic field embedded in a non-magnetic atmosphere. The magnetic field vector and filling factor are reliable inferred in only $50 \%$ based on the regular profiles. Therefore, $12 \%$ of the field of view harbour hG fields with filling factors typically below $30 \%$. At our present spatial resolution, $70 \%$ of the pixels apparently are non-magnetised.
\end{abstract}

Key words. Sun: atmosphere - Sun: magnetic fields - techniques: polarimetric - methods: observational

\section{Introduction}

At any given time, even at the maximum of the Sun's activity cycle, most of the solar surface is covered by areas of low average magnetic fluxes. These areas are called the quiet Sun. Within the quiet Sun, we refer to the network as the reticular pattern at the border of supergranular cells, which has magnetic fluxes of $\sim 10^{18}-10^{19}$ Mx (e.g. Stenflo 1973; Martínez González et al. 2012a). The interiors of these cells are permeated by a weaker magnetism whose fluxes are lower by one or two orders of magnitude. We call these regions the very quiet Sun. Recently, evidence of even more quiet areas within the very quiet Sun has been reported, the so-called dead calm areas, where magnetic fluxes are the weakest detected $\left(\sim 10^{15} \mathrm{Mx}\right.$; Martínez González et al. 2012b).

Very sensitive spectro-polarimeters are needed to detect the very faint polarisation signals (more than two orders of magnitude weaker than those from active regions) of the very quiet areas of the Sun. In addition, high spatio-temporal resolution observations are needed to properly study the small-scale dynamic 
magnetism of these regions. The spectro-polarimeter of the Hinode satellite (Lites et al. 2013), the Imaging Magnetograph eXperiment (IMaX, Martínez Pillet et al. 2011) instrument onboard the Sunrise mission (Solanki et al. 2010), and the Tenerife Infrared Polarimeter (Collados et al. 2007) using the adaptive optics system (Berkefeld et al. 2010) of the German Vacuum Tower Telescope (Observatorio del Teide) have provided such data during the past decade (e.g. Khomenko et al. 2003; López Ariste et al. 2006; Orozco Suárez et al. 2007; Lites et al. 2008; Martínez González et al. 2008c; Danilovic et al. 2010; Quintero Noda et al. 2013; Requerey et al. 2015, and references therein). This explains why the magnetism of the quiet Sun has received increasing attention in the past few yrs. This is also partly motivated by the exceptionally extended minimum following solar cycle 23 (2007-2011).

When analysing the statistical properties of polarimetric signals, most of the works in the literature agree on the picture of the very quiet Sun magnetism: the signals are the same everywhere in the solar disc. In other words, the quiet Sun looks the same regardless of the observer's line of sight (Martínez González et al 2008b; Lites et al. 2008; Orozco Suárez \& Katsukawa 2012). Moreover, their magnetic properties either do vary with the activity cycle, or this variation is very weak (Faurobert et al. 2001; Buehler et al. 2013; Lites et al. 2014; Faurobert \& Ricort 2015). Finally, the degree of spatial coherence of the signals increases with the polarimetric signal. By this we mean that the weakest detected fluxes of the quiet Sun on scales below $1000 \mathrm{~km}$ show a very dynamic, intermittent, stochastic, magnetic activity unlike the "deterministic" characterisation of the magnetic structure of sunspots and other active structures formed by the socalled flux tubes (López Ariste et al. 2006; Martínez González et al. 2010b; Stenflo 2010). Stronger fluxes, but still two orders of magnitude weaker than those of the network regions, may organise at granular scales and form magnetic loops (e.g. Martínez González et al. 2007; Centeno et al. 2007; Gömöry et al. 2010; Ishikawa et al. 2010) that appear intermittently on the quiet Sun (Martínez González et al. 2012b) and have energetic implications on higher layers (Martínez González et al. 2010a). Small-scale magnetic flux tubes have also been inferred with high-precision spectropolarimetry and high spatial resolution data (Lagg et al. 2010; Requerey et al. 2014).

Advancing our knowledge of the very quiet Sun magnetism requires interpreting polarimetric signals in terms of physical quantities. To do so, we rely on inversion codes that search for the best model parameters that fit the observed profiles. The model needs to be selected so that the residual of the fit and the observations is uncorrelated noise. This is where many works in the literature disagree and disputed results appear. An early controversy revolved around the strength of magnetic fields. While works in the near-infrared inferred hG fields (e.g. Khomenko et al. 2003; Martínez González et al. 2008c), other works used visible lines and derived $\mathrm{kG}$ fields (e.g. Socas-Navarro \& Sánchez Almeida 2002; Domínguez Cerdeña et al. 2003). Later, the Hinode satellite provided spectro-polarimetric data in the visible with improved spatial resolution. In these data, $\mathrm{hG}$ were found in the internetwork (e.g. Orozco Suárez et al. 2007). Today, most of the community agrees that magnetic fields in the very quiet Sun are in the hG regime or even weaker. However, although one controversy was solved, the analysis of Hinode data has recently instigated another controversy on the inclination of magnetic fields: while some works point towards a mostly isotropic distribution (Martínez González et al. 2008b; Lites et al. 2008; Asensio Ramos 2009; Stenflo 2010), others claim that the fields are mostly horizontal (Orozco Suárez \& Katsukawa 2012; Orozco Suárez \& Bellot Rubio 2012; Bellot Rubio \& Orozco Suárez 2012). We still do not fully understand the reasons behind these disagreements. Some works claim that the observed profiles have not enough information to constrain model parameters (Martínez González et al. 2006), and some others show that the photon noise can introduce biases and degeneracies in the inversion problem (Asensio Ramos 2009; Stenflo 2010; Borrero \& Kobel 2012).

This paper presents high-precision observations with high spatial resolution of the very quiet Sun with two Zeeman-sensitive Fe I lines at $1565 \mathrm{~nm}$. The line at $1564.8 \mathrm{~nm}$ has a Landé factor of 3 . This, together with its H-band wavelength, turns it into one of the most sensitive lines to polarisation, and one of the best lines to measure magnetic fields in the Zeeman regime within the reach of current instrumentation. The observations were performed with the new German GREGOR telescope, achieving an unprecedented spatial resolution at $1.5 \mu \mathrm{m}$ of $0.4^{\prime \prime}$. In this work, we analyse these highquality data to shed some light on the apparent controversy surrounding the topology of the magnetic field in the very quiet Sun.

\section{Observations}

On 2015 September 17 we recorded $1.5 \mu \mathrm{m}$ spectro-polarimetric data of a quiet region at disc centre, with a spectral sampling of $40.1 \mathrm{~m} \AA$. We used the Tenerife Infrared Polarimeter working with the the GRegor Infrared Spectrograph (Collados et al. 2007, 2012) installed at the German GREGOR telescope (Schmidt et al. 2012). The adaptive optics system (Berkefeld et al. 2012) was locked on granulation and provided a spatial resolution of $\sim 0.4^{\prime \prime}$, as inferred from the power spectrum of the continuum image. We scanned an area of $61.6^{\prime \prime} \times 13.5^{\prime \prime}$, using a time integration of $4.6 \mathrm{~s}$ per slit position (taking into account overheads, the cadence was $\sim 8 \mathrm{~s}$ ) and a step size of $0.135^{\prime \prime}$. The data set used in this work is the same as in Lagg et al. (2016).

The data were corrected for flatfield and bias and were demodulated with the dedicated software (Schlichenmaier \& Collados 2002). Additional corrections were applied to match the continuum of the atlas by Livingston \& Wallace (1991) following a similar strategy as in Allende Prieto et al. (2004). We modelled the average quiet-Sun intensity profile as the addition of the atlas convolved with a Gaussian function and a flat spectrum, whose weight is the so-called white-light veil. We iteratively fitted a high-order polynomial, the white-light veil, and the width of the Gaussian to the average quiet-Sun intensity profile. The Gaussian width was consistent with the spectral resolution of the instrument, and the veil was about $9 \%$ (similar to the value obtained by Lagg et al. 2016). We applied the veil correction only to the intensity spectra, and we divided all Stokes parameters by the inferred high-order polynomial.

We then used the principal component analysis (Loève 1955; Rees \& Guo 2003) to minimise the uncorrelated Gaussian noise. This technique consists of constructing a base of eigenvectors from the covariance matrix of the data. The very few first eigenvectors contain most of the correlation and the rest contain basically uncorrelated noise. Therefore, we can reconstruct the data with the truncated base of eigenvectors that contain useful information and remove uncorrelated noise (see the application of this technique for denoising solar and stellar spectra in e.g. Martínez González et al. 2008c,a). To ensure that we did not lose any physical information, we reconstructed the data with 41 eigenvectors. Unpolarised and polarised interference fringes 

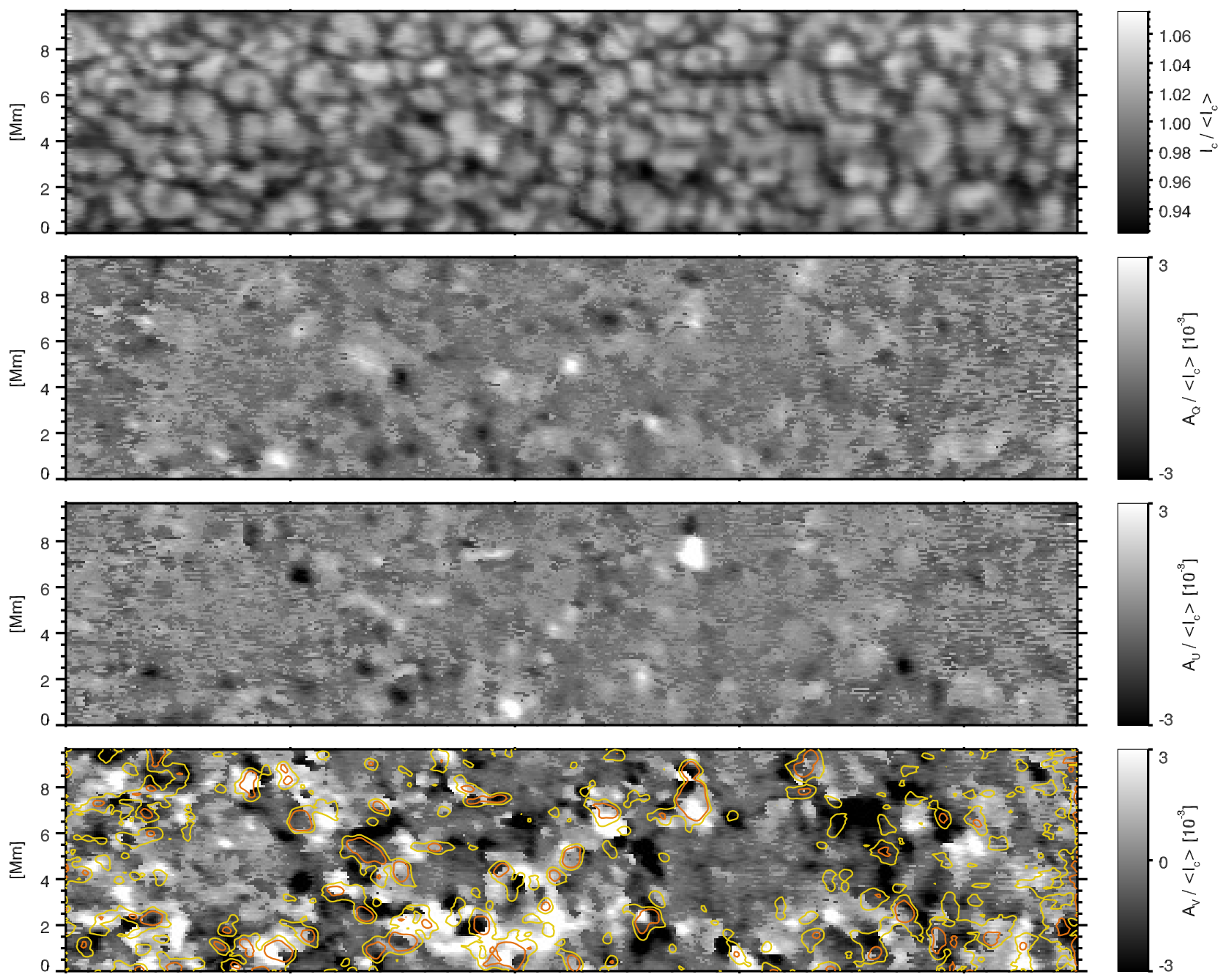

Fig. 1. Intensity and polarisation maps of the observed quiet region at disc centre. The yellow and orange contours display the linear polarisation $\sqrt{Q^{2}+U^{2}}$ with amplitude levels of $1 \times 10^{-3}\left\langle I_{\mathrm{c}}\right\rangle$ and $1.5 \times 10^{-3}\left\langle I_{\mathrm{c}}\right\rangle$, respectively. Although the defined linear polarisation is a biased quantity, these contours are reliable since their values are more than one order of magnitude above the noise level. The amplitude of the circular polarisation has been computed as the highest value of the amplitudes of the two lobes, and the sign is given by the blue lobe. The amplitude of a lobe is calculated as the average value in two pixels around the maximals. The amplitudes of Stokes $Q$ or $U$ are calculated as the average in two pixels around the position of the maximum, taking the absolute value of the parameters.

(Semel 2003) are evident in the eigenvectors of all the Stokes parameters. We removed them in the truncated base of eigenvectors by fitting sinusoidal functions. Other artefacts, such as jumps created by the CCD separations, were also removed from the eigenvectors. After those corrections, we re-orthogonalised the truncated base of eigenvectors using the Gram-Schmidth algorithm. After the PCA denoising, the noise level in polarisation is $\sigma_{\mathrm{n}}=10^{-4}\left\langle I_{\mathrm{c}}\right\rangle$, with $\left\langle I_{\mathrm{c}}\right\rangle$ the continuum intensity averaged over the field of view (FOV). The polarisation continuum was set to zero, with a precision given by the noise level.

Figure 1 displays the intensity and polarisation maps after our denoising procedure, showing the high quality of the GRIS data. The circular and linear polarisation signatures permeate the quiet Sun: $96 \%$ of the observed area has either linear or circular polarisation amplitudes above $4 \sigma_{\mathrm{n}}$. Interestingly, $74 \%$ of the observed area has linear (either Stokes $Q$ or $U$ ) polarisation and circular polarisation above $4 \sigma_{\mathrm{n}}$. The spatial distribution of Stokes $V$ or Stokes $Q$ or $U$ are very similar in the sense that the stronger signals form a filamentary pattern with dead calm areas of $\sim 10^{\prime \prime}$ diameter ( $40 \mathrm{Mm}^{2}$ area) that contain the weaker signals.
The yellow and orange contours overplotted on the circular polarisation in Fig. 1 represent the linear polarisation $\left(\sqrt{Q^{2}+U^{2}}\right)$ with $1 \times 10^{-3}\left\langle I_{\mathrm{c}}\right\rangle$ and $1.5 \times 10^{-3}\left\langle I_{\mathrm{c}}\right\rangle$, respectively. Most of the linear polarisation links circular polarisation regions with opposite polarities, but does not form simple $\Omega$ shaped loops. We obtained simpler structures when we adopted a higher threshold for the linear polarisation. Taking only the orange contours into account, we count 47 loop-like structures, which gives an emergence rate of 1.7 loop $\operatorname{arcsec}^{-2} \mathrm{~h}^{-1}$. This rate has been calculated as the number of loops divided by the scan area and the loop lifetimes. The lifetime of a loop is defined as the time in which a loop is recognised as two opposite polarities linked by linear polarisation. This lifetime is $\sim 2 \mathrm{~min}$ and has been derived from previous works by Martínez González \& Bellot Rubio (2009) and Martínez González et al. (2012b). This emergence rate is safely computed from raster scans when the exposure time is much shorter than the loop lifetimes. When the exposure time is of the order of the loops lifetime, the emergence rate is overestimated (e.g. the value of 0.3 loop $\operatorname{arcsec}^{-2} \mathrm{~h}^{-1}$ that can be obtained using previous $1^{\prime \prime}$ TIP-II data, as were 
Table 1. Free parameters used in models $1 \mathrm{C}$ and $2 \mathrm{C}$.

\begin{tabular}{cccccccccccccccccc}
\hline \hline & $T^{1}$ & $v_{\mathrm{LOS}}{ }^{1}$ & $B^{1}$ & $\theta^{1}$ & $\phi^{1}$ & $v_{\mathrm{mac}}{ }^{1}$ & $v_{\mathrm{mic}}{ }^{1}$ & $T^{2}$ & $v_{\mathrm{LOS}}{ }^{2}$ & $B^{2}$ & $\theta^{2}$ & $\phi^{2}$ & $v_{\mathrm{mac}}{ }^{2}$ & $v_{\mathrm{mic}}{ }^{2}$ & $\alpha$ & $\alpha_{\mathrm{SL}}$ \\
\hline $1 \mathrm{C}$ & 5 & 1 & - & - & - & 1 & 1 & $5^{1}$ & 1 & 1 & 1 & 1 & $1^{2}$ & 1 & 1 & - \\
$2 \mathrm{C}$ & 5 & 1 & 1 & 1 & 1 & 1 & 1 & $5^{1}$ & 1 & 1 & 1 & 1 & $1^{2}$ & 1 & 1 & $30 \%$ \\
\hline
\end{tabular}

Notes. The superscripts 1 and 2 represent the first and second component. The variables are the temperature $T$, the LOS velocity $v_{\text {LOS }}$, the strength $B$, inclination $\theta$, and azimuth $\chi$ of the magnetic field, the macroturbulent velocity $v_{\text {mac }}$, the microturbulent velocity $v_{\text {mic }}$, the filling factor $\alpha$, and the fraction of stray light $\alpha_{\mathrm{SL}} \cdot{ }^{(1)}$ The temperature at the continuum of the second component is forced to be the same as in the first component. (2) The macroturbulent velocity of the second component is forced to be the same as the first component.

presented in Martínez González et al. 2007). The present emergence rate is almost seven times higher than the rate inferred from IMaX data using the Fe I visible line at $525 \mathrm{~nm}$ at $\sim 0.15^{\prime \prime}$ (0.25 loop $\operatorname{arcsec}^{-2} \mathrm{~h}^{-1}$ Martínez González et al. 2012b).

\section{Model approaches to infer the physical parameters}

A critical point in any inference problem is selecting the parametric model that is used to reproduce the observations. In our data, about $48 \%$ of Stokes $V$ profiles are not regular and have only one or more than two lobes. Stokes $V$ signals also exhibit area and amplitude asymmetries. In many cases, the linear and circular Stokes profiles are not mutually compatible. The lineof-sight (LOS) velocity inferred from Stokes $Q$ and $U$ profiles is different from that inferred from Stokes $V$. This incompatibility also occurs with regard to the width of the lines. Furthermore, there are instances in which the Stokes parameters cannot be reproduced with a single magnetic field vector. All together, these are indicators that both the magnetic field and the LOS velocity show gradients within the resolution element, that is, along the optical depth and/or across the surface. Given the richness and complexity of the data, we interpret them in terms of three different models. We invert our spectro-polarimetric data using the Stokes Inversion based on Response functions code (Ruiz Cobo $\&$ del Toro Iniesta 1992).

We first adopted a model in which the resolution element is shared by a magnetic and a non-magnetic atmosphere. The nonmagnetic component was assumed to contain the contribution of instrumental unpolarised stray light as well as possible magnetic substructure, that is, mixed polarities or extremely weak fields that have no net contribution to the polarised spectrum. The magnetic atmosphere was assumed to have a constant magnetic field vector and velocity along the LOS. The temperature stratification in both atmospheres was modified starting from the original VAL-C (Vernazza et al. 1981) model with a maximum of five nodes, forcing the temperature of the continuum to be the same in both components. The microturbulence, macroturbulent velocity, and the LOS velocity are free height-independent parameters in both components. The filling factor $\alpha$, that is, the fraction of the resolution element occupied by the magnetic atmosphere, is also a free parameter. The total number of free parameters in this model is 18 . This model allowed us to reproduce the general properties of two-lobed Stokes $V$ profiles with low area and amplitude asymmetries and with compatible linear polarisation profiles. It also allows the comparison with previous studies with high Zeeman sensitivity. In the following, we refer to this model as model $1 \mathrm{C}$.

Secondly, we modelled the Stokes profiles with two magnetic atmospheres and a global, unpolarised stray-light component (model 2C). The magnetic field vector of the two components was assumed constant, as well as the LOS velocity. The temperature stratifications in the two components were variable and had a maximum of five nodes. We only constrained the temperature of the two components at the continuum to be the same. In this model, the global stray-light profile is a synthetic profile (to avoid correlation between the observed profiles through fringes and other systematics) obtained as the best fit to the average Stokes $I$ in the FOV. The filling factor of the stray-light component was fixed to $30 \%$ and was estimated from the absorption of the $\pi$ component in the umbral core of sunspot observations (see Borrero et al. 2016). Since this value is approximate, we checked that a percentage of the stray-light factor from $20 \%$ to $60 \%$ did not significantly change the results. The model has 21 free parameters. We are aware that some of the complexity in the Stokes profiles that this model attempts to reproduce may be produced by local polarised stray light. We do not exactly know the level of this stray light, and since we do not study particular patches but the statistics in the FOV, we did not attempt to model it. A list of the free parameters used in models $1 \mathrm{C}$ and $2 \mathrm{C}$ can be found in Table 1.

\section{One magnetic structure embedded in a field-free atmosphere}

To have a general view of the quiet-Sun magnetism, Fig. 2 displays the maps of the inferred magnetic field strength $B$, the magnetic field inclination $\theta$, the magnetic field azimuth $\chi$, and the filling factor using a single height-independent magnetic atmosphere (model 1C). Most of the area is full of weak hG fields, although there are a few patches with $\mathrm{kG}$ fields (white contours). These $\mathrm{kG}$ patches coincide with vertical fields, but only the largest patch (at $[20,1.5] \mathrm{Mm})$ has filling factors higher than $15 \%$. In general, vertical fields correspond to stronger field strengths, while the more horizontal fields have strengths of $\sim 150 \mathrm{G}$. This behaviour is the same as that observed by Martínez González et al. (2008c) with the same instrument but with lower spatial resolution.

In $49 \%$ of the FOV, model $2 \mathrm{C}$ yields a fit of similar quality as model $1 \mathrm{C}$ for Stokes $Q, U$, and $V$, simultaneously. In this case, we prefer the model with fewer free parameters. From these pixels, we selected those with amplitudes larger than $4 \sigma_{n}$, with two-lobed Stokes $V$ profiles. For these pixels, which account for $23 \%$ of the FOV, the magnetic flux density $\phi=B \alpha \cos \theta$ is well constrained by the observed profiles. The magnetic flux density is defined through the solar surface, in our case, the normal to the surface coincides with the LOS. We note that at the disc centre, the magnetic flux density only depends on the LOS magnetic field, that is, on the Stokes $V$ profile.

We performed several inversions at each pixel in which we fixed the inclination to values from 5 to $85 \mathrm{deg}$ and let the code find a solution with the field strength, the filling factor, and the microturbulent velocity as free variables. We defined 

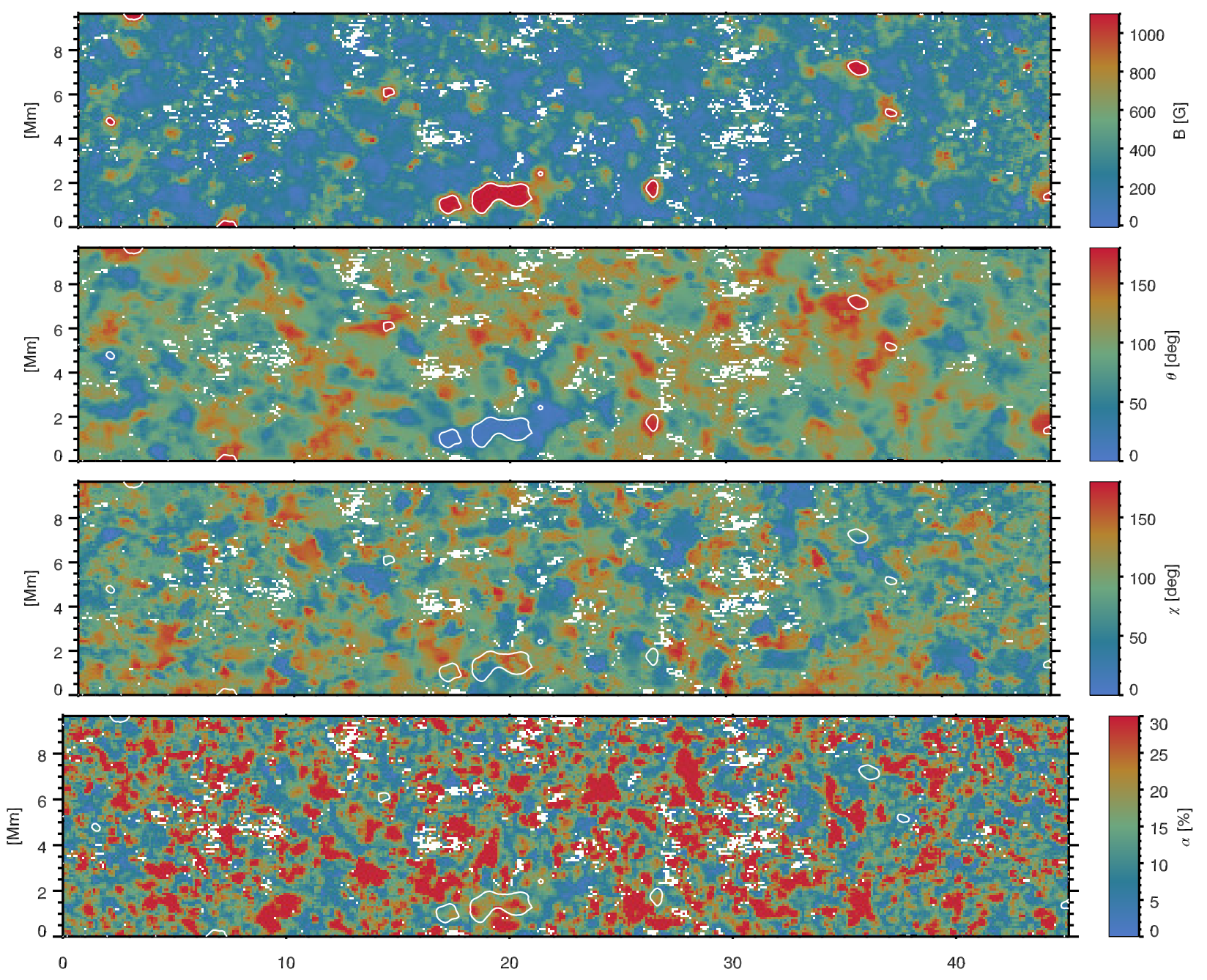

$[\mathrm{Mm}]$

Fig. 2. Maps of the inferred magnetic field vector (strength, inclination, and azimuth) and the filling factor of the magnetic component. The white contours denote the areas with $\mathrm{kG}$ fields. White pixels contain signal below $4 \sigma_{\mathrm{n}}$ and hence are not inverted.

the normalised rms of the polarisation profiles for each inversion with a fixed value of the inclination as

$\sum_{S=Q U V} \frac{\sigma\left(S^{\mathrm{fit}}-S^{\mathrm{obs}}\right)}{3 \sigma_{\mathrm{n}}}$.

The symbol $\sigma$ stands for the standard deviation operation, $S^{\text {obs }}$ for the observed polarisation profiles, and $S^{\text {fit }}$ for its best fit. When the normalised $\mathrm{rms}$ in these inversions varies by more than $20 \%$ (and the individual values are below 1.2, i.e., reasonable fits), we considered the magnetic field vector to be well constrained by the observations, since the inversion code is not able to reproduce the observations equally well with different inclination values. The pixels with regular Stokes $V$ profiles, where the magnetic field is reliably recovered using a single atmosphere approach, represent $12 \%$ of the FOV.

Figure 3 shows the histograms of the magnetic flux density for the pixels in which the magnetic field vector is not reliably inferred (solid line) and for those where it is well constrained by the observations (dotted line). We find stronger fluxes for the latter pixels.

The histograms of the magnetic field vector and the filling factor of the pixels where they are well constrained by the observations are displayed in Fig. 4. Most of the fields are of the order of hG: $80 \%$ of the pixels have strengths below $450 \mathrm{G}$

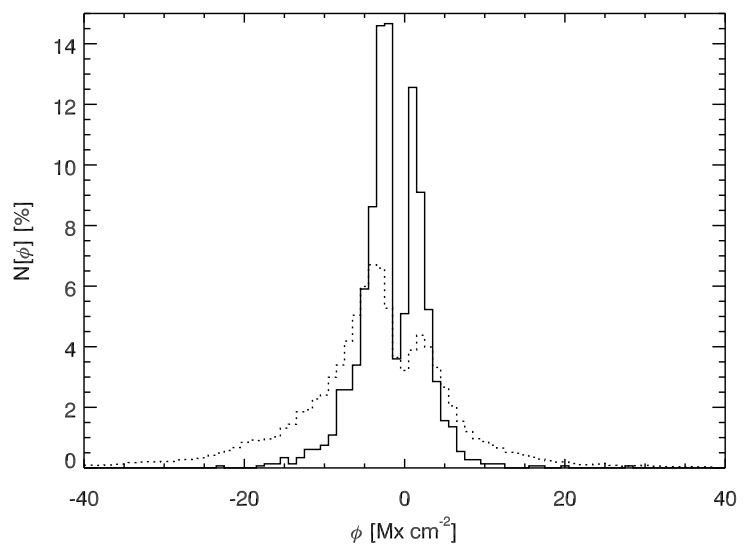

Fig. 3. Histograms of the magnetic flux density inferred using a model with one magnetic atmosphere embedded in a field-free volume. The histograms represent the inversions at those pixels where Stokes $Q$ or $U$ or $V$ are above $4 \sigma_{\mathrm{n}}$, model $1 \mathrm{C}$ is preferred by the observations, and the Stokes $V$ is a two-lobed profile. The solid line represents the values of the magnetic flux density for those pixels where the magnetic field strength, inclination, and filling factor are not reliably retrieved. The dotted line displays the histogram for those pixels where the magnetic field vector, inclination, and azimuth are well constrained by the observations. 

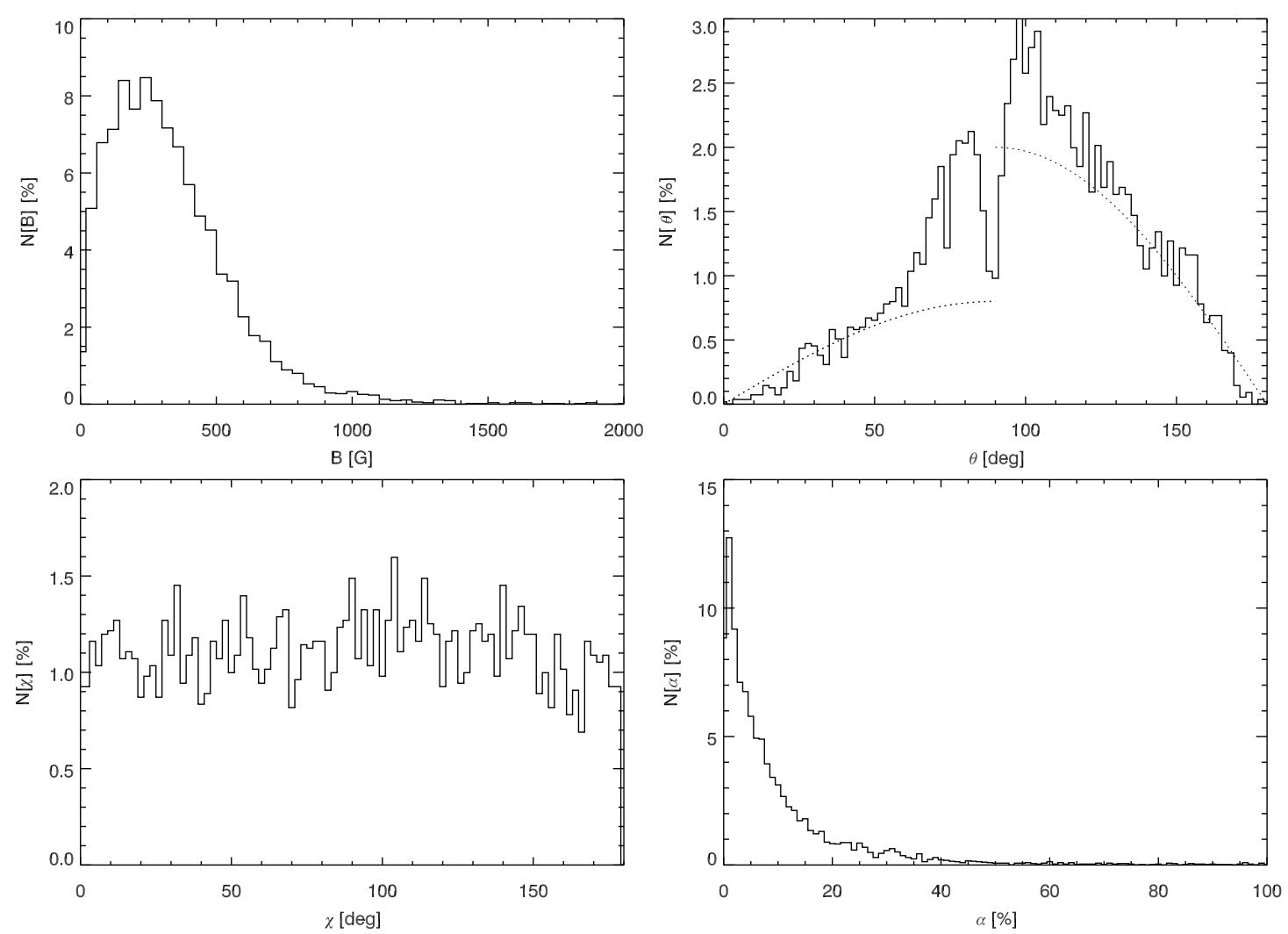

Fig. 4. Histograms of the inferred field vector (strength, inclination, and azimuth) and the filling factor of the magnetic component using a model with one magnetic atmosphere embedded in a field-free volume. The histograms represent the inversions at those pixels where Stokes $Q$ or $U$ or $V$ are above $4 \sigma_{\mathrm{n}}$, the Stokes $V$ is a two-lobed profile, and the field vector and filling factor are well constrained by the observed profiles.

and $50 \%$ are below $250 \mathrm{G}$. The field strength distribution has a peak at $\sim 250 \mathrm{G}$. These results for the field strength are compatible with previous studies using near-infrared data at lower resolution and longer exposure times (Khomenko et al. 2003; Martínez González et al. 2008c). However, the peak at $250 \mathrm{G}$ was located at $\sim 450 \mathrm{G}$ in these studies. Lowering the spatial resolution or increasing the exposure time dilutes the spectropolarimetric signals and results in a higher detection limit for the field strength.

The inclination distribution (top right panel in Fig. 4) has an excess of horizontal $\left(70^{\circ}-110^{\circ}\right)$ fields with respect to an isotropic distribution (that follows $p(\theta)=\sin \theta$ and $p(\chi)=1$ ). Surprisingly, the flux in the two polarities is not balanced in the observed FOV. Previous studies of very quiet regions reported a polarity balance, even with smaller observed areas (e.g. Lites 2002; Domínguez Cerdeña et al. 2003; Martínez González et al. 2008c). This imbalance occurs for all signals, hence it cannot be a result of unipolar network patches or a unipolar weak component previously undetected.

\section{Two magnetic atmospheres and stray light}

In $51 \%$ of the FOV, the reduced chi-square of Stokes $Q, U$, and $V$ of the inversions with model $2 \mathrm{C}$ was smaller than in model $1 \mathrm{C}$ inversions. Figure 5 shows three examples in which the fit with two magnetic components is better than the fit from a single magnetic component and a non-magnetic atmosphere. In the top panel, the Stokes $V$ profile is well fitted by the two models, but for Stokes $Q$ and $U$ the single magnetic component fails to reproduce the amplitudes and their Doppler shift. The middle and bottom panels represent irregular Stokes $V$ profiles that cannot be fitted with a single magnetic field; they require a more complex model with two magnetic components.
The results of the inversions for those pixels that are fitted better by model $2 \mathrm{C}$ are presented in Fig. 6 . The large (small) component is defined as the one with the larger (lower) filling factor. The two components have clearly different magnetic properties. The larger component has weak field strengths, $80 \%$ are below $90 \mathrm{G}$ and $50 \%$ are below $50 \mathrm{G}$, the inclination distribution has a peak at $90^{\circ}$, and the azimuth has a uniform distribution. The small component has stronger fields, for which $80 \%$ are below $750 \mathrm{G}$ and $50 \%$ are below $350 \mathrm{G}$, and it is almost isotropic, with a slight polarity imbalance.

Figure 7 displays the inferred inclination and filling factor values versus the magnetic field strength. The large component (shown in blue) has an almost constant field strength for inclinations between $50^{\circ}$ and $130^{\circ}$. The more vertical fields (around $30^{\circ}$ and $150^{\circ}$ ) have slightly stronger, but still very weak field strengths $(\sim 100-150 \mathrm{G}$ on average). The more horizontal fields of the small component have the weakest fields, and the more vertical are stronger, reaching values above $1 \mathrm{kG}$. The relationship of the filling factor and the field strength is compatible with a hyperbola given by $B \approx 5200 / \alpha[\%]$ (dotted line) for fields below $300 \mathrm{G}$. This behaviour, and the field strengths involved, led us to assume that there is not enough information in the polarised spectra of the weakest signals to reliably infer the field strength. In these cases, the only quantity that could be inferred without ambiguity was the magnetic flux density. Above $300 \mathrm{G}(70 \%)$, field strength, inclination, and filling factor are unambiguous.

To check the reliability of the magnetic parameters inferred for the large component, we performed the following numerical test. We selected 1000 pixels from the sample used for Fig. 6 with inclinations of the large component between 80 and $100 \mathrm{deg}$. We note that half the area of the distribution has 

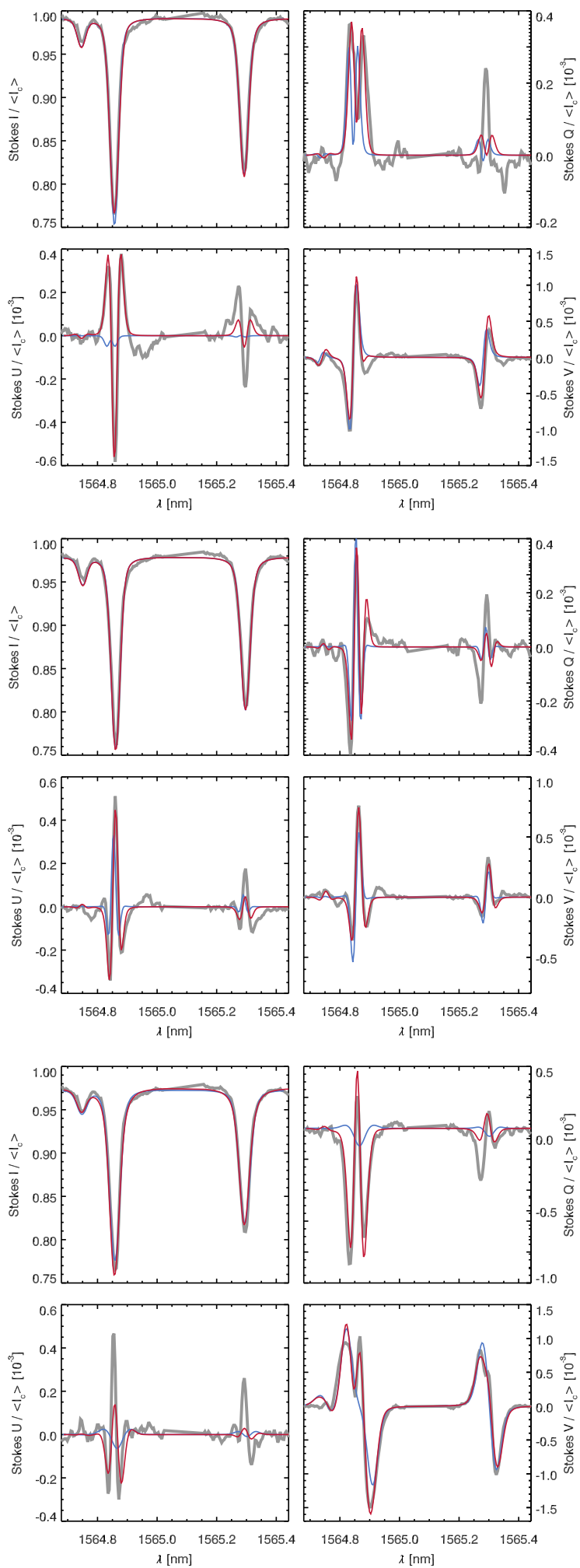

Fig. 5. Three typical examples of Stokes profiles, displayed as the thick grey line. The blue line represents the inversion with the model that considers only one magnetic component embedded in a non-magnetised atmosphere. The red line displays the inversion using a model with two magnetic atmospheres coexisting with the $30 \%$ contamination of unpolarised stray light.

inclinations between 80 and $100 \mathrm{deg}$. The remaining pixels that are not as horizontal are very likely in the first order weakfield regime of the Zeeman effect given their extremely low field strengths. This regime implies that the field strength, inclination, and filling factor are degenerated quantities. For the low field strengths, the only way to avoid the weak-field regime is to have a field inclined enough to generate linear polarisation above the noise level. For this reason, we performed the reliability test for the more inclined fields of the large component.

We inverted the more horizontal pixels of the large component by forcing the inclination from $5 \mathrm{deg}$ to $85 \mathrm{deg}$ (or 100 to $175 \mathrm{deg}$ for the opposite polarity) and letting the code modify only the magnetic field strength and the microturbulent velocity of the large component. The atmospheric parameters of the small component, the percentage of stray light, and the filling factor of the large component were fixed. The top left panel in Fig. 8 shows the normalised rms of the Stokes profiles with respect to the difference between the fixed inclination and the one inferred from the $2 \mathrm{C}$ model. The flat curve in this figure indicates that the code is able to find a good fit for all values of the inclination differences by modifying the magnetic field strength. The microturbulent velocity has always reasonable values below $2 \mathrm{~km} \mathrm{~s}^{-1}$. The variation of the field strength as compared to the one inferred from the $2 \mathrm{C}$ inversion (top right panel in Fig. 8) can reach values close to $100 \%$ for inclination differences near $90 \mathrm{deg}$. This means that with the present spatial resolution and polarimetric sensitivity, there is not enough information in the spectral lines to infer the field strength and the inclination of the large component independently. We have checked that the only quantity that remains the same in all inversions with fixed inclinations and hence is the only one that can be reliably inferred for the large component is the magnetic flux density, defined as $B \alpha \cos \theta$. The histogram of the magnetic flux density of the large component is shown in the leftmost panel of Fig. 9. It shows more extended wings than a Gaussian, and has an average flux density of $-0.84 \pm 0.02 \mathrm{Mx} \mathrm{cm}^{-2}$ (arrow).

To check the reliability of the magnetic parameters inferred for the small component, we performed the following test. We fixed the inclination of the small component from 5 to $85 \mathrm{deg}$ (or 100 to $175 \mathrm{deg}$ ) and let the code vary the field strength and the microturbulent velocity of the small component, and the field strength, inclination, and microturbulent velocity of the large component, as well as the filling factor. We distinguished between three populations of the small component: weak fields (below $250 \mathrm{G}$ ), strong fields (between 250 and $800 \mathrm{G}$ ), and very strong fields (above $800 \mathrm{G}$ ). The middle left panel of Fig. 8 shows the normalised rms of the Stokes profiles with respect to the difference between the fixed inclination and the one inferred from the $2 \mathrm{C}$ model. For the weak fields, the code is able to reproduce the Stokes profiles regardless of the inclination by varying mainly the field strength of the small component. The variation of the field strength of the small component with respect to the 2C inversion can be as huge as $100 \%$ for the cases in which the inclination difference is largest (close to $90 \mathrm{deg}$ ). We note that the behaviour of the relative error of the magnetic field strength of the small component is very similar to the one for the large component in the previous test. Similarly as for the large component, this means that with the present spatial resolution and polarimetric sensitivity, there is not enough information in the spectral lines to infer the field strength and the inclination of the small component for fields below $250 \mathrm{G}$. The only quantity that remains the same in all inversions with fixed inclinations is again the magnetic flux density. The histogram of the magnetic flux density of the small component for fields below $250 \mathrm{G}$ is shown in the middle panel of Fig. 9. It shows more extended wings than a Gaussian, and contains slightly more strong fluxes than the large component. It has an average flux density of $-1.8 \pm 0.02 \mathrm{Mx} \mathrm{cm}^{-2}$ (arrow). 

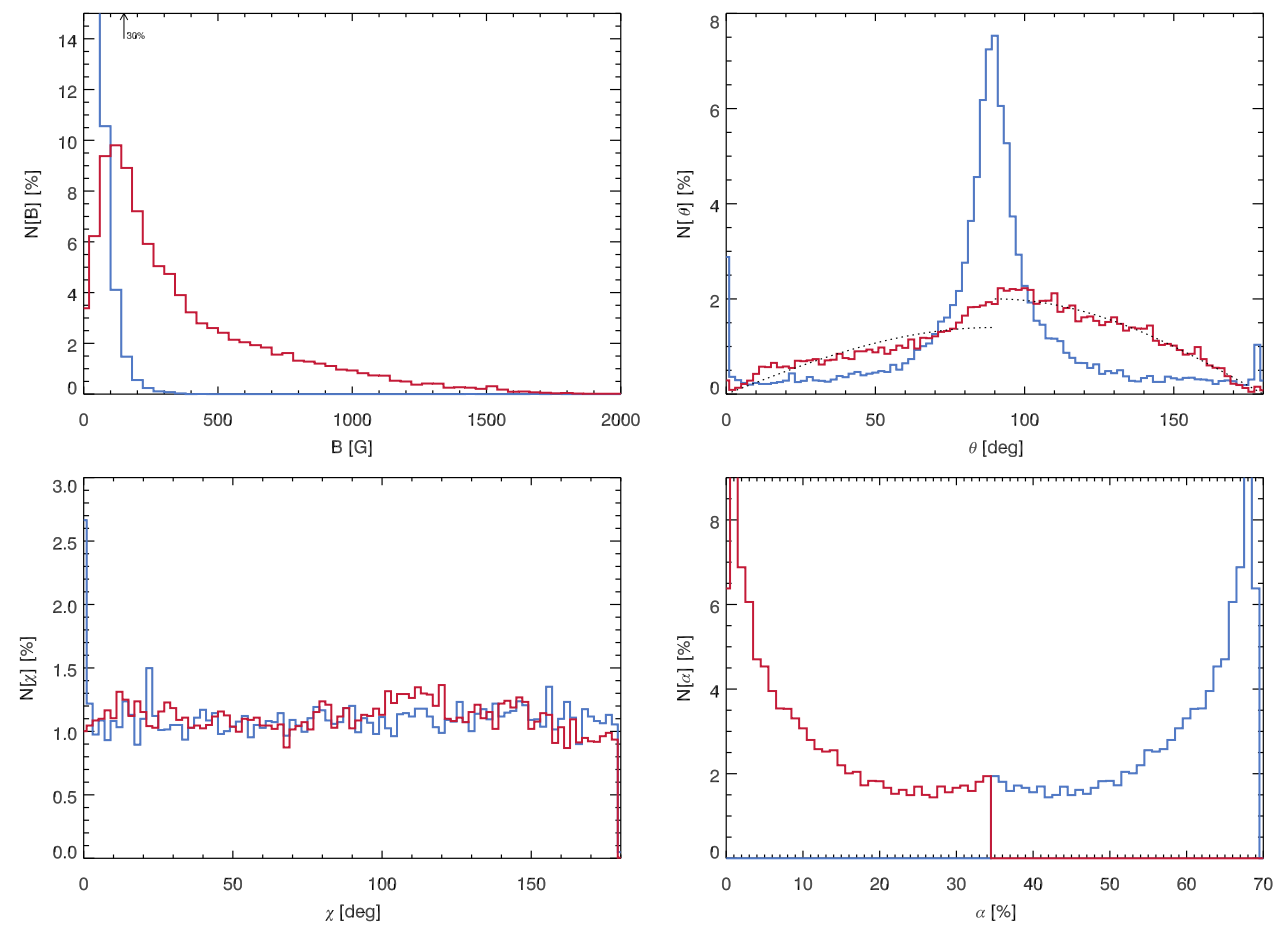

Fig. 6. Histograms of the inferred field vector (strength, inclination, and azimuth) and the filling factors using a model with two magnetic atmospheres contaminated by $30 \%$ of unpolarised stray light. All the inverted pixels have Stokes $Q$ or $U$ or $V$ signals above $4 \sigma_{\mathrm{n}}$ and have better fits than the single magnetic component inversion. The blue (red) lines represent the large (small) component, the one with larger (smaller) filling factor. The dotted grey lines superposed to the inclination histogram represent a $\sin \theta$ distribution. Taking into account that the azimuth is uniform, these dotted lines represent an isotropic distribution of the field vector.
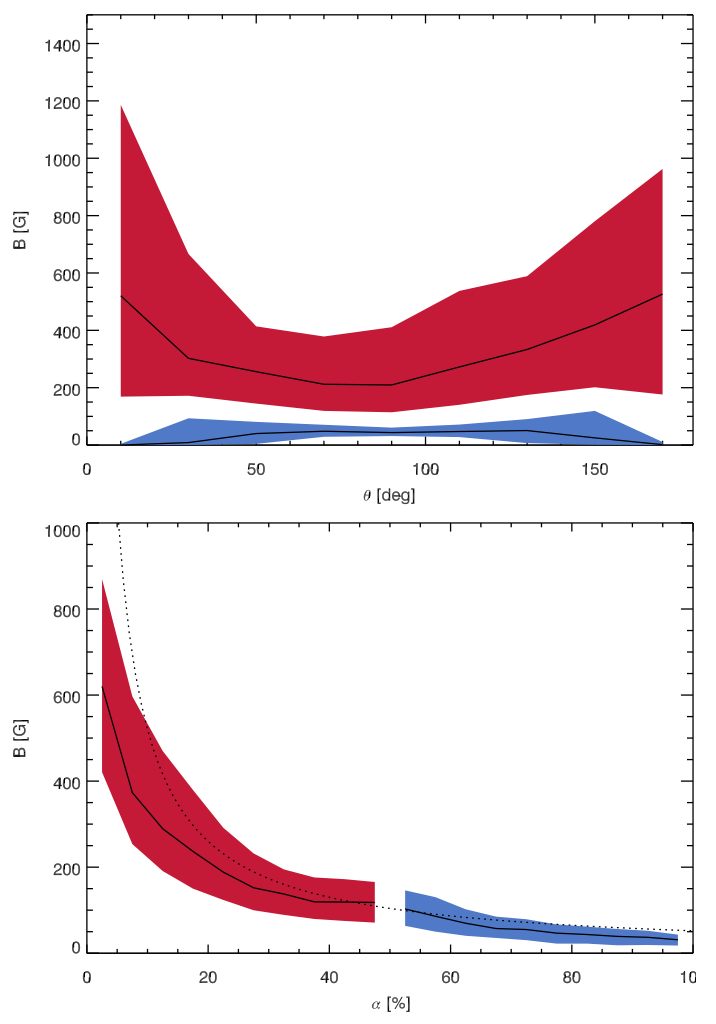

Fig. 7. Magnetic field strength versus inclination (top) and versus filling factor (bottom). All the inverted pixels have Stokes $Q$ or $U$ or $V$ signals above $4 \sigma_{\mathrm{n}}$. Blue (red) represents the large (small) component, the one with larger (smaller) filling factor. The black line represents the median value, and the shaded areas enclose percentiles 25 to 75 . The dotted black line is an hyperbolic relationship given by $5200 / \alpha[\%]$.
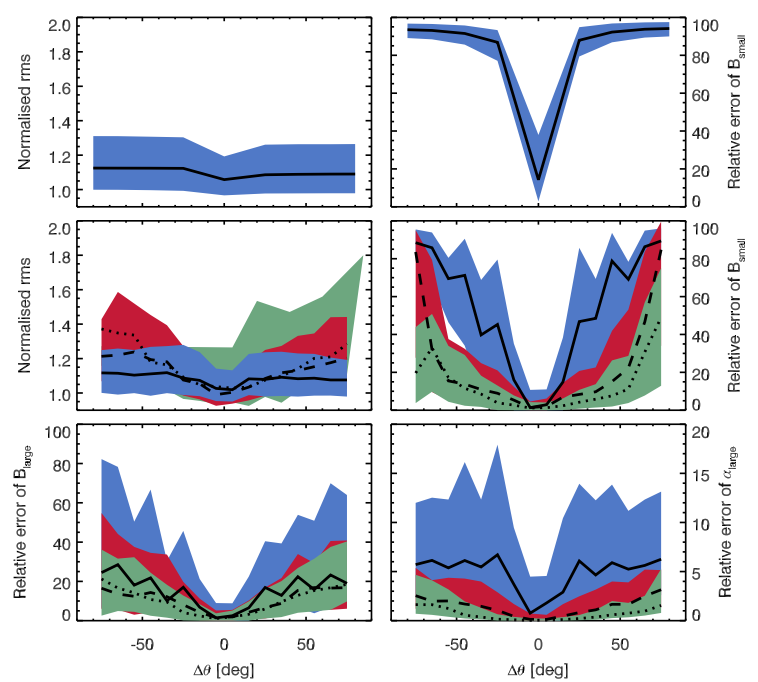

Fig. 8. Top panels: results of the numerical test to check the validity of the inferred magnetic properties of the large component. The top left panel represents the the normalised rms of the polarisation profiles defined as $\sum_{S=Q U V} \sigma\left(S^{\mathrm{fit}}-S^{\mathrm{obs}}\right) / 3 \sigma_{\mathrm{n}}$ plotted versus the inclination we have forced in each inversion. The top right panel shows the relative modification of the magnetic field strength of the large component to account for the forced values of the inclination. The black line represents the 50 percentile (median value), and the shaded blue areas enclose percentiles 25 to 75 . The remaining panels show the numerical test to check the reliability of the small component. The middle left panel represents the normalised $\mathrm{rms}$ of the polarisation profiles for the weak population (solid line for the median and blue shaded areas for percentiles 25 to 75), the strong population (dashed line and red shaded areas), and the very strong population (dotted line and green shaded areas). The middle and bottom right panels show the relative modification of the magnetic field strength of the small component and the filling factor to account for the forced values of the inclination. 

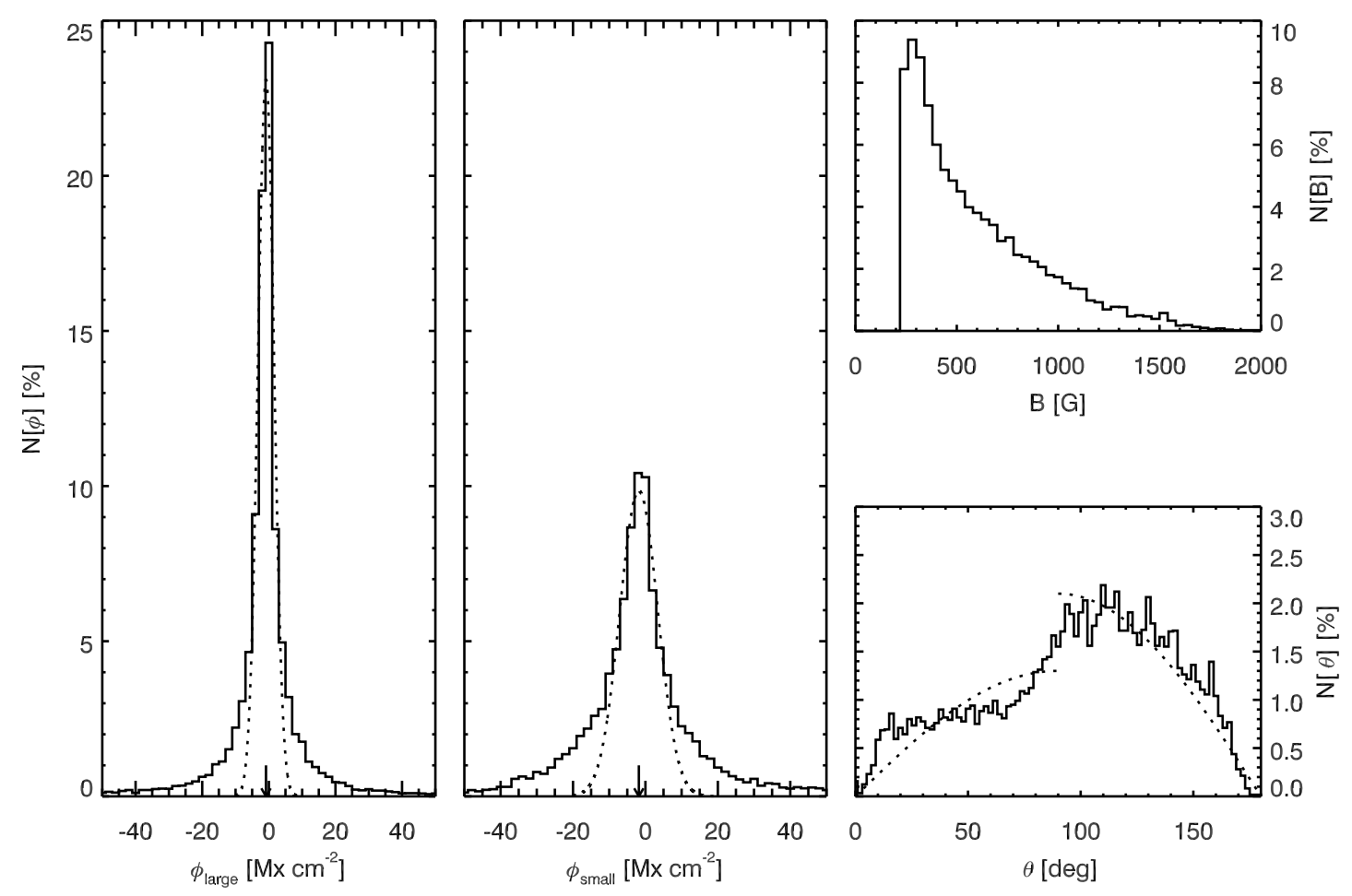

Fig. 9. Left panel: histogram of the magnetic flux density inferred for the large component. Middle panel: histogram of the magnetic flux density of the small component for those pixels with fields strengths below $250 \mathrm{G}$. The vertical arrow marks the average value of the fitted Gaussian core (dotted line). The top and bottom right panels display the magnetic field strength and inclination of the small component for field strengths above $250 \mathrm{G}$.

For the strong and very strong fields, the code is not able to reproduce the Stokes profiles for all the fixed inclinations equally well. It is only capable of obtaining similar fits when the inclination difference is smaller than around $30 \mathrm{deg}$. It can also be seen that the relative error of the magnetic field strength of the small component is almost constant around $10 \%$. This means that the Zeeman splitting is already important, or that the linear polarisation is well above the noise level, or both, preventing the code from finding a good fit by varying the field strength if we fix an inclination different than the one inferred from the $2 \mathrm{C}$ inversion. Therefore, we conclude that the magnetic field vector and filling factor of the small component are constrained by the observations for fields above $250 \mathrm{G}$. The leftmost panels of Fig. 9 display the magnetic field strength and inclination of the small component that are reliably inferred. The results are very similar to those in Fig. 6. The azimuth and the filling factor are not displayed, but they are almost equal to those in Fig. 6. The field strengths have a distribution whose decay resembles an exponential function with an average field of $390 \mathrm{G}$. The inclination still shows a clear polarity imbalance. Together with the uniform azimuth, the topology is close to isotropic. As inferred from the validity test, the inclination has an uncertainty of about $30 \mathrm{deg}$, the field strength about $10 \%$, and the filling factor about $1 \%$.

Asymmetries and single or additional lobes in Stokes $V$, or the relative velocities between Stokes $V$ and the linear polarisation profiles, can also be explained by gradients along the optical depth. To check if the observations can discern vertical and horizontal gradients, we performed an inversion with a magnetic component (with a filling factor of $70 \%$ ) with allowed gradients of all the physical properties along the optical depth, except for the microturbulent velocity (model $\mathrm{G}$ ). The remaining $30 \%$ is filled with the same unpolarised stray-light component used in the $2 \mathrm{C}$ inversions. Allowing gradients of the physical quantities along the LOS is the only way to reproduce the area asymmetry in Stokes $V$ (Solanki 1993).

Inversions with model $\mathrm{G}$ are difficult to be performed automatically to the whole map because different observed profiles require a different number of nodes in the stratification of the physical quantities. To check if the observations are better reproduced by vertical or horizontal gradients, we selected some representative profiles. To invert these selected examples, we proceeded by fixing the number of nodes of the temperature at a maximum of 5. For the remaining physical quantities, we first tried with two nodes (a linear gradient) in magnetic field strength and LOS velocity, and then increased the complexity until we found a good fit. The number of free parameters needed to fit the representative profiles was between 20 (only linear gradients) up to 42 when more complicated gradients were allowed. We are aware that such large number of free parameters cannot be constrained by the observations and multiple solutions to the inverse problem can exist (Asensio Ramos et al. 2012). But this study is beyond the scope of this paper. Our aim here is not to extract real stratifications of the very quiet atmosphere but to see how observations are reproduced with this model $\mathrm{G}$ as compared to model 2C.

Figure 10 displays the three selected representative profiles to perform the inversions with model G. We chose profiles that have some features that show the need for a more complex scenario than a single magnetic field in the resolution element. The profiles in the top left panel are representative of those pixels with regular polarisation signatures (Stokes $Q$ and $U$ also have the same Doppler shift as Stokes $V$ ) with area and amplitude asymmetries. In this case, we obtain a similar fit with model G (red line) and model 2C. Moreover, it is not surprising that a good fit was also obtained with just one constant magnetic 

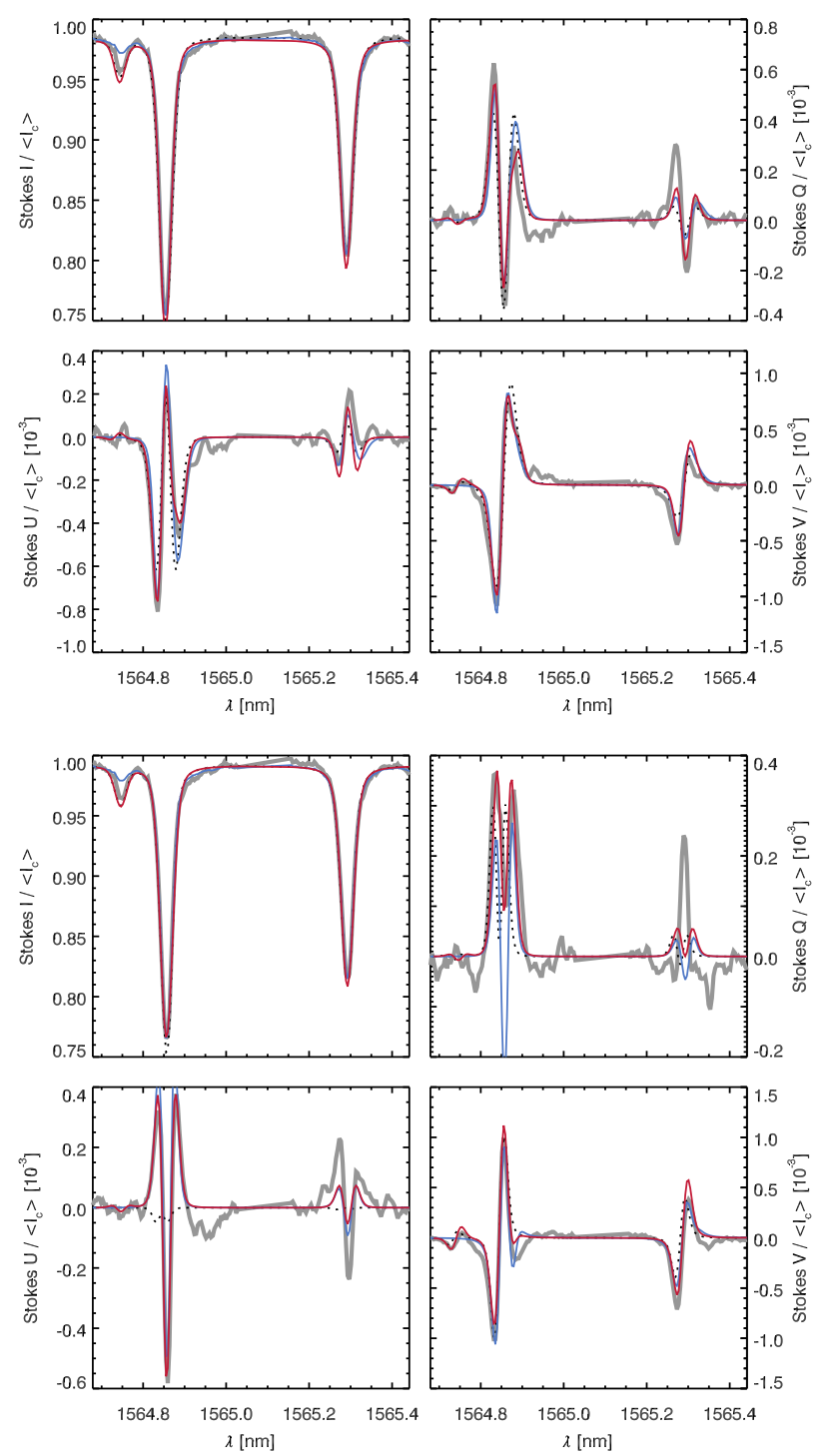
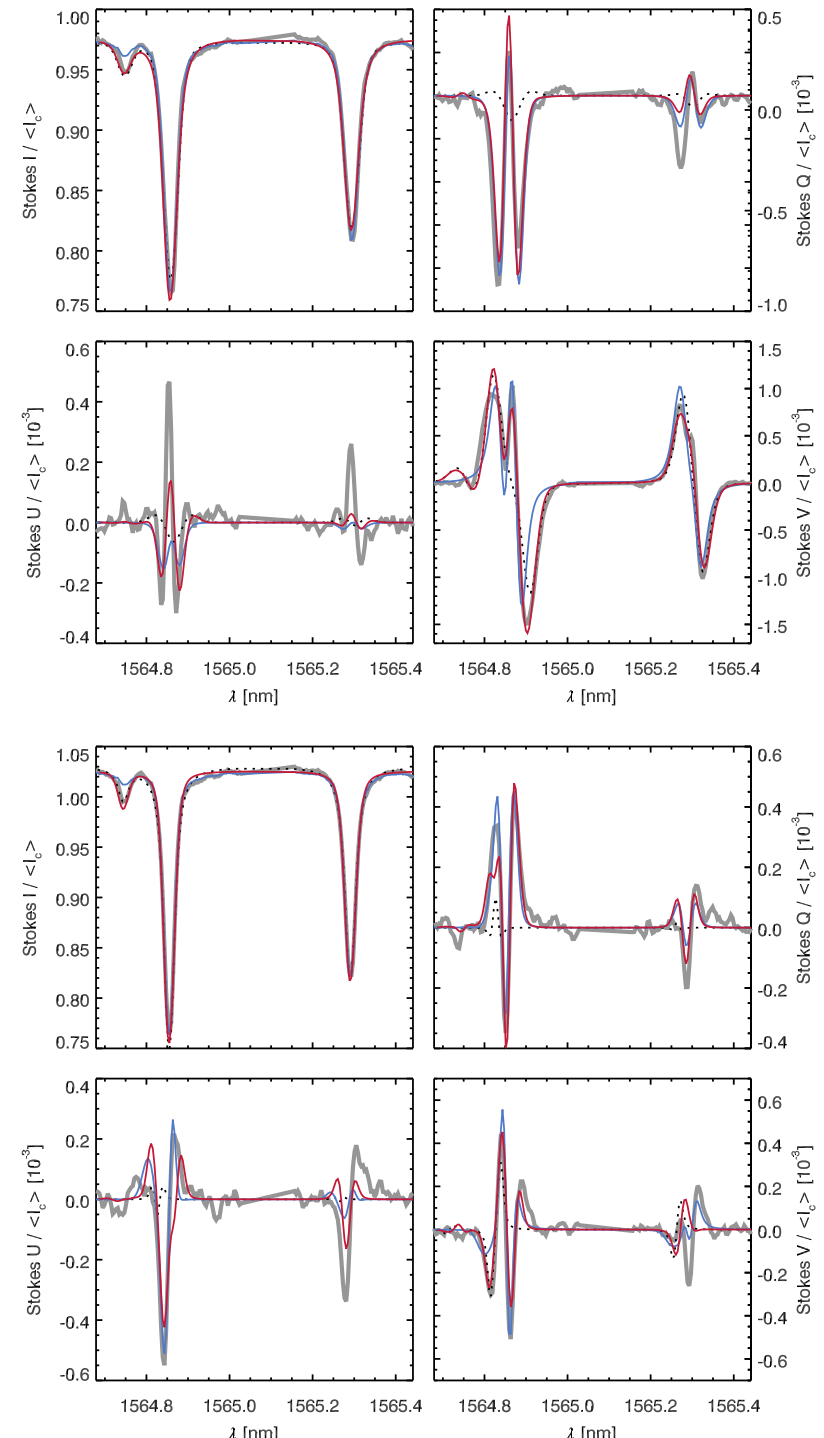

Fig. 10. Four typical examples of Stokes profiles, displayed as the thick grey line. The blue line represents the inversion using a model with two magnetic atmospheres (with constant field vector and LOS velocity) coexisting with the $30 \%$ contamination of unpolarised stray light. The red line display the inversion of one magnetic atmosphere coexisting with the $30 \%$ contamination of unpolarised stray light with allowed gradients in all physical quantities (except for the microturbulent velocity). The dotted black line represents the inversion with the model that considers only one magnetic component (with constant field vector and LOS velocity) embedded in a non-magnetised atmosphere.

component (model 1C; dotted black line), since the asymmetries are not large.

The top right and bottom panels show clear examples in which Stokes $Q$ and $U$ are not compatible with Stokes $V$. The top right panel shows a better fit in Stokes $V$ for model $G$ than for model $2 \mathrm{C}$. However, similar fits for model $\mathrm{G}$ and $2 \mathrm{C}$ are obtained in the bottom panels. In these types of profiles, it is clear that the observations are better fitted with magnetic substructure within the resolution element than with a constant magnetic field (model 1C). However, since the area asymmetries are not very large, the observations do not have the information necessary to distinguish between gradients along the LOS (model G) or across the surface (model 2C).

\section{Conclusions and discussion}

We have presented $1.56 \mu \mathrm{m}$ spectropolarimetry with high spatial resolution $\left(0.4^{\prime \prime}\right)$ and high polarimetric sensitivity $\left(10^{-4}\left\langle I_{\mathrm{c}}\right\rangle\right)$ of the quietest areas of the Sun. A visual inspection of the data revealed that most of the polarisation profiles show clear indications of magnetic substructure within the resolution element, that is, of relative velocities between the Stokes parameters, area, and amplitude asymmetries in Stokes $V$, and/or irregular Stokes $V$ profiles. This substructure can be due to gradients of the physical properties along the LOS (note that Stokes $V$ area asymmetries can only be obtained with vertical gradients) or across the solar surface. Horizontal gradients can be of solar origin (true substructure within the resolution element) or due to stray light in the telescope, or both. To properly take the stray light into account, we need to know the telescope point spread function. At present, this function is not very well characterised, and we preferred to use an approximation of the stray light as an unpolarised quiet-Sun spectrum with a constant contribution in the FOV.

Half of our observed quiet-Sun region is better explained by magnetic substructure within the resolution element. However, 
we cannot distinguish whether this substructure comes from gradients of the physical parameters along the LOS or from horizontal gradients (across the surface). From a model with two magnetic components (plus a 30\% stray-light contamination), we defined the large (small) population as the one with larger (smaller) filling factor. After a numerical test, we reached the conclusion that the magnetic field strength, inclination, and filling factor of the large component are degenerate and that we can only rely on the magnetic flux density. We also showed that the strength, inclination, and filling factor of the small component are constrained by the observations for fields above $250 \mathrm{G}$ : they are isotropic, hG fields. We found a polarity imbalance that occurs for all inclinations. To confirm the solar origin of this imbalance requires more quiet-Sun observations.

The other half of the observed quiet-Sun area can be explained by a single magnetic field embedded in a non-magnetic atmosphere. From these pixels, $\sim 50 \%$ are two-lobed Stokes $V$ profiles. This means that $23 \%$ of the FOV can be adequately explained with a single constant magnetic field embedded in a non-magnetic atmosphere. The others are irregular, very weak profiles, hence the improvement in the fit using a two-component model is negligible. From the regular profiles, the magnetic field vector and filling factor were reliable inferred only in $50 \%$. We inferred hG fields with small filling factors $(89 \%$ are below 30\%); at our present spatial resolution, $70 \%$ of the pixel is apparently non-magnetised, however. There is growing evidence that this large fraction of the resolution element is magnetised, but at much smaller scales than our present resolution capabilities. From the theoretical point of view, modern magnetohydrodynamical simulations show a myriad of magnetic fields tangled at scales as small as a few km (e.g. those analysed by Lagg et al. 2016). From the observational point of view, each time we improve our resolution capabilities, we detect weaker and smaller-scale fields. In addition, Hanle measurements (e.g. Trujillo Bueno et al. 2004) claim that there is much more magnetic energy in a pixel than is detected by the Zeeman effect.

Acknowledgements. The authors are very grateful to an anonymous referee that helped to improve the manuscript and to strengthen the results and to $\mathrm{L}$. R. Bellot Rubio for fruitful discussions on the subject of this paper. This work is based on observations made with the German GREGOR telescope at the Spanish Observatorio del Teide of the Instituto de Astrofísica de Canarias. The 1.5-m GREGOR solar telescope was built by the German consortium under the leadership of the Kiepenheuer-Institut für Sonnenphysik with the LeibnizInstitut für Astrophysik Potsdam, the Institut für Astrophysik Göttingen, the Max-Planck-Institut für Sonnensystemforschung in Göttingen, and the Instituto de Astrofísica de Canarias, and with contributions by the Astronomical Institute of the Academy of Sciences of the Czech Republic. The GRIS instrument was developed thanks to the support by the Spanish Ministry of Economy and Competitiveness through the project AYA2010-18029 (Solar Magnetism an Astrophysical Spectropolarimetry). Financial support by the Spanish Ministry of Economy and Competitiveness and the European FEDER Fund through projects AYA2014-60476-P and AYA2014-60833-P are gratefully acknowledged. Financial support by Consolider-Ingenio 2010 CSD2009-00038 is also acknowledged. A.A.R. acknowledges financial support through the Ramón y Cajal fellowship. SJGM is grateful for financial support from the Leibniz Graduate School for Quantitative Spectroscopy in Astrophysics, a joint project of the Leibniz Institute for Astrophysics Potsdam and the Institute of Physics and Astronomy of the University of Potsdam. This paper made use of the IAC Supercomputing facility HTCondor (http://research.cs.wisc.edu/ htcondor/).

\section{References}

Allende Prieto, C., Asplund, M., \& Fabiani Bendicho, P. 2004, A\&A, 423, 1109 Asensio Ramos, A. 2009, ApJ, 701, 1032

Asensio Ramos, A., Manso Sainz, R., Martínez González, M. J., et al. 2012, ApJ, 748, 83

Bellot Rubio, L. R., \& Orozco Suárez, D. 2012, ApJ, 757, 19
Berkefeld, T., Soltau, D., Schmidt, D., \& von der Lühe, O. 2010, Appl. Opt., 49, 155

Berkefeld, T., Schmidt, D., Soltau, D., von der Lühe, O., \& Heidecke, F. 2012, Astron. Nachr., 333, 863

Borrero, J. M., \& Kobel, P. 2012, A\&A, 547, A89

Borrero, J. M., Asensio Ramos, A., et al. 2016, A\&A, 596, A2 (GREGOR SI)

Buehler, D., Lagg, A., \& Solanki, S. K. 2013, A\&A, 555, A33

Centeno, R., Socas-Navarro, H., Lites, B., et al. 2007, ApJ, 666, L137

Collados, M., Lagg, A., Díaz Garcí, A., J. J., et al. 2007, in The Physics of Chromospheric Plasmas, eds. P. Heinzel, I. Dorotovič, \& R. J. Rutten, Heinzel, ASP Conf. Ser., 368

Collados, M., López, R., Páez, E., et al. 2012, Astron. Nachr., 333, 872

Danilovic, S., Beeck, B., Pietarila, A., et al. 2010, ApJ, 723, L149

Domínguez Cerdeña, I., Sánchez Almeida, J., \& Kneer, F. 2003, A\&A, 407, 741 Faurobert, M., \& Ricort, G. 2015, A\&A, 582, A95

Faurobert, M., Arnaud, J., Vigneau, J., \& Frisch, H. 2001, A\&A, 378, 627

Gömöry, P., Beck, C., Balthasar, H., et al. 2010, A\&A, 511, A14

Ishikawa, R., Tsuneta, S., \& Jurčák, J. 2010, ApJ, 713, 1310

Khomenko, E. V., Collados, M., Solanki, S. K., Lagg, A., \& Trujillo Bueno, J. 2003, A\&A, 408, 1115

Lagg, A., Solanki, S. K., Riethmüller, T. L., et al. 2010, ApJ, 723, L164

Lagg, A., Borrero, J. M., Asensio Ramos, A., M. Collados, M., et al. 2016, A\&A, 596, A6 (GREGOR SI)

Lites, B. W. 2002, ApJ, 573, 431

Lites, B. W., Kubo, M., Socas-Navarro, H., et al. 2008, ApJ, 672, 1237

Lites, B. W., Akin, D. L., Card, G., et al. 2013, Sol. Phys., 283, 579

Lites, B. W., Centeno, R., \& McIntosh, S. W. 2014, PASJ, 66, 4

Livingston, W., \& Wallace, L. 1991, An atlas of the solar spectrum in the infrared from 1850 to $9000 \mathrm{~cm}^{-1}$ (1.1 to 5.4 micrometer)

Loève, M. M. 1955, Probability theory (Princeton: Van Nostrand Company)

López Ariste, A., Ramírez Vélez, J. C., Tomczyk, S., Casini, R., \& Semel, M. 2006, in Solar Polarization Workshop 4, eds. R. Casini, \& B. W. Lites, ASP Conf. Ser., 358, 54

Martínez González, M. J., Collados, M., \& Ruiz Cobo, B. 2006, A\&A, 456, 1159

Martínez González, M. J., Collados, M., Ruiz Cobo, B., \& Solanki, S. K. 2007, A\&A, 469, L39

Martínez González, M. J., Asensio Ramos, A., Carroll, T. A., et al. 2008a, A\&A, 486, 637

Martínez González, M. J., Asensio Ramos, A., López Ariste, A., \& Manso Sainz, R. 2008b, A\&A, 479, 229

Martínez González, M. J., Collados, M., Ruiz Cobo, B., \& Beck, C. 2008c, A\&A, 477, 953

Martínez González, M. J., \& Bellot Rubio, L. R. 2009, ApJ, 700, 1391

Martínez González, M. J., Manso Sainz, R., Asensio Ramos, A., \& Bellot Rubio, L. R. 2010a, ApJ, 714, L94

Martínez González, M. J., Manso Sainz, R., Asensio Ramos, A., López Ariste, A., \& Bianda, M. 2010b, ApJ, 711, L57

Martínez González, M. J., Bellot Rubio, L. R., Solanki, S. K., et al. 2012a, ApJ, 758, L40

Martínez González, M. J., Manso Sainz, R., Asensio Ramos, A., \& Hijano, E. 2012b, ApJ, 755, 175

Martínez Pillet, V., Del Toro Iniesta, J. C., Álvarez-Herrero, A., et al. 2011, Sol. Phys., 268, 57

Orozco Suárez, D., \& Bellot Rubio, L. R. 2012, ApJ, 751, 2

Orozco Suárez, D., \& Katsukawa, Y. 2012, ApJ, 746, 182

Orozco Suárez, D., Bellot Rubio, L. R., del Toro Iniesta, J. C., et al. 2007, ApJ, 670, L61

Quintero Noda, C., Martínez Pillet, V., Borrero, J. M., \& Solanki, S. K. 2013, A\&A, 558, A30

Rees, D., \& Guo, Y. 2003, in Solar Polarization, eds. J. Trujillo-Bueno, \& J. Sanchez Almeida, ASP Conf. Ser., 307, 85

Requerey, I. S., Del Toro Iniesta, J. C., Bellot Rubio, L. R., et al. 2014, ApJ, 789, 6

Requerey, I. S., Del Toro Iniesta, J. C., Bellot Rubio, L. R., et al. 2015, ApJ, 810, 79

Ruiz Cobo, B., \& del Toro Iniesta, J. C. 1992, ApJ, 398, 375

Schlichenmaier, R., \& Collados, M. 2002, A\&A, 381, 668

Schmidt, W., von der Lühe, O., Volkmer, R., et al. 2012, Astron. Nachr., 333, 796

Semel, M. 2003, A\&A, 401, 1

Socas-Navarro, H., \& Sánchez Almeida, J. 2002, ApJ, 565, 1323

Solanki, S. K. 1993, Space Sci. Rev., 63, 1

Solanki, S. K., Barthol, P., Danilovic, S., et al. 2010, ApJ, 723, L127

Stenflo, J. O. 1973, Sol. Phys., 32, 41

Stenflo, J. O. 2010, A\&A, 517, A37

Trujillo Bueno, J., Shchukina, N., \& Asensio Ramos, A. 2004, Nature, 430, 326

Vernazza, J. E., Avrett, E. H., \& Loeser, R. 1981, ApJS, 45, 635 